\title{
Estudio de la frecuencia die hemoglobinopatías en las islas de San Andrés y Providencia, Colombia
}

\author{
María del Pilar Bernal ${ }^{1}$, Alejandro Giraldo², Antonio J. Bermúdez ${ }^{1}$, Edith Moreno ${ }^{3}$
}

\begin{abstract}
Resumen
Se realizó un estudio destinado a determinar la frecuencia de hemoglobinopatías en las islas caribeñas de San Andrés y Providencia, en 544 individos individuos: 443 de San Andrés y 101 de Providencia. La frecuencia en San Andrés fue del 12,8\% y en Providencia 20,8\%; las hemoglobinopatías más frecuentes fueron: el rasgo falciforme, la hemoglobina $\mathrm{C}$ y la beta-talasemia. Los datos indican, que las hemoglobinopatías en esta región del país como problema de salud pública, tienen importancia y nos dan información sobre el origen geográfico de la población.
\end{abstract}

\begin{abstract}
Summary
In order to determine the frequency of hemoglobinopathies in the Colombian Caribbean Islands, a population study was done in 544 persons, 443 from San Andrés and 101 from Providencia with frequencies of $12.8 \%$ and $20.8 \%$ respectively. The hemoglobinic disorders more frequently found weres HBS, HBC and $\beta$-thalassemia. The results obtained showed that hemoglobinopahies in this region represent a public health problem and reveai the origin of the population.
\end{abstract}

El estudio de las hemoglobinopatías tiene varios puntos de interés, como son: el aspecto clínico, ya que son la causa de enfermedades hemolíticas y otros síndromes patológicos; genético, porque son marcadores de identificación de individuos y familias; y, antropológico, porque aporta información de comparación étnica y de la influencia migratoria que ha sufrido una población determinada (1). Dentro de las variantes hemoglobínicas, las de mayor importancia clínica son la hemoglobina S cuando se presenta en forma homocigota y la talasemia mayor, ya sea alfa $(\alpha)$ o beta $(\beta)$. Las manifestaciones clínicas de la anemia falciforme causada por la $\mathrm{HbS}$, se presentan con un espectro muy amplio: desde crisis dolorosas

\footnotetext{
1 Grupo de Genética, INS.

2 Laboratorio Nacional de Salud Samper Martínez, INS.

3 Grupo de Química Clínica, INS.
}

hasta complicaciones serias y la muerte, dependiendo en parte de las condiciones ambientales. El rasgo drepanocítico (AS) es de naturaleza benigna; sin embargo, han sido informadas algunas anormalidades clínicas con complicaciones vaso-oclusivas durante períodos de hipoxia, presentándose el fenómeno drepanocítico e infartos esplénicos a altitudes superiores de $6.500 \mathrm{msnm}$. El mantenimiento del polimorfismo para $\mathrm{HbS}$ a lo largo del mundo se ha asociado con la protección contra Plasmodium falciparum (2, 3), ocurriendo lo mismo con las talasemias. La hemoglobina $\mathrm{C}$ en estado de homocigosis presenta una patología leve comparada con la anterior, y los portadores son individuos cuyo fenotipo hematológico puede ser normal o presentar algunas características típicas.

En el grupo de las talasemias, en donde existe una deficiencia parcial o total de las cadenas alfa c beta de la molécula, la patología depende del 
grado de síntesis de dichas cadenas, siendo desdeasintomáticos para individuos heterocigotos hasta presentar el síndrome de hidropesía fetal que corresponde al estado de homocigoto de $\alpha$ talasemia.

Para la mayoría de las hemoglobinopatías se ha determinado el origen geográfico; es el caso de las $\mathrm{HbS}$ y $\mathrm{HbC}$, con mutaciones en la cadena $B$, $B S\left(B 6^{\text {Glu_Val }}\right)$ y $B C\left(B^{\text {Glu_Lys }}\right)$, cuyo origen es africano: la $\mathrm{HbC}$ de Africa occidental (2). Se ha determinado a través de estudios de haplotipos del gen de ß-globina, que la mutación que origina la $\mathrm{HbS}$, ocurrió en forma independiente en cuatro poblaciones africanas: Algeria (Africa norte), Bantú (Africa central), Senegal (Atlántico africano) y en Benin (centro-oeste africano) (2-5). Las talasemias $\alpha$ y $\beta$ tienen una distribución geográfica mucho más amplia a través de Africa, Europa y Asia (3, 7).

\section{Materiales y métodos}

Los individuos participantes en el estudio fueron seleccionados por los censos estatales de que dispone el servicio de salud del departamento; la selección fue aleatoria según la ubicación de las manzanas y las casas: aquéllas dispuestas en la mitad de la cuadra más la subsiguiente y las de enfrente, para un total de 1.000 individuos seleccionados. Estos fueron informados por diferentes medios y se incluyeron a quienes participaban voluntariamente, quedando los 544 individuos.

Se tomaron $5 \mathrm{~mL}$ de sangre total con EDTA de cada individuo y para cada una de las muestras se realizó biometría que incluyó $\mathrm{RCB}, \mathrm{Hb}$, hematocrito, VCM, $\mathrm{HCM}, \mathrm{CHCM}$; extracción de hemoglobina y electroforesis en membranas de acetato de celulosa a pH básico $(8,9)$; cuantificación de $\mathrm{Hb} \alpha 2$ porel método de elución (9); cuantificación de $\mathrm{Hb}$ fetal por denaturalización alcalina $(10,11)$; inducción de drepanocitos con metabisulfito de sodio (12, 13); estabilidad al isopropanol (14); y electroforesis en agar citrato $\mathrm{pH}$ ácido (15).

\section{Resultados y discusión}

De las 544 muestras analizadas de las dos islas, 77 de ellas presentaron hemoglobinas anormales (14,3\%), distribuyéndose así: 57 casos son positivos en San Andrés (12,8\%) y 21 en Providencia $(20,8 \%)$ (tabla 1). Los fenotipos de hemoglobinopatías halladas en las dos islas fueron: el rasgo falciforme o HBA-S y que representan el mayor grupo con frecuencia de $6,8 \%$, el rasgo $\mathrm{A}-\mathrm{C}$ con $2,9 \%$, el doble heterocigoto $\mathrm{S} /$ $B^{+}$Tal con $2,0 \%$ y los portadores de $B^{+} T a l$ con $1,7 \%$. Solamente en San Andrés se halló persistencia hereditaria de hemoglobina festa (PHHF) con frecuencia de $0,7 \%$, un individuo con doble variante hemoglobínica S-C, y 1 homocigoto S$\mathrm{S}$, o anemia falciforme (tabla 2).*

Es de anotar que no existen estudios previos al presente en las islas de San Andrés y Providencia. Si comparamos los datos obtenidos con los de otros estudios realizados en el país (tabla 3), se puede ver que la distribución es muy variada. Estas investigaciones son las informadas hasta el momento.

En el estudio de Restrepo publicado en 1970, no se encuentra ningún desorden en hemoglobinas en población indígena de algunas tribus del Chocó (1.422 individuos) y $2,2 \%$ en población mixta y pacientes con indicación de estudio de desórdenes de la hemoglobina en la ciudad de Medellín (2.817 individuos), resultando en una frecuencia promedio de $7,6 \%$ para el estudio, cifra sugerida como valor promedio para el resto del país (15). Las hemoglobinopatías informadas fueron: A-S $4,3 \%, A-C 1,4 \%, S-C 0,3 \%$, S-S 0, $2 \%, C-C 0,03 \%$, A-D 0,15\%, A-B+Tal 0,65\%, S-B+Tal 0,13\%, C$B+$ Tal $0,03 \%, \mathrm{PHHF} 0,04 \%$, otras variantes $0,04 \%$.

El estudio de Espinel (16) informa una frecuencia de $3,8 \%$ para población negra de un total de 1.043 individuos estudiados distribuidas en los fenotipos A-C 2,11\%, A-S 1,4\%, S-S 0,1\% y S-C $0,2 \%$; mientras que en un grupo de indígenas Waunana del Chocó, de 52 individuos, la frecuencia alcanzó el 21,2\% únicamente de heterocigotos A-C; dicha población está ubicada frente a Docordó, una población de negros.

En la investigación de Fonseca (17), se encontró una frecuencia de $0,6 \%$ en 300 individuos de población de origen cundiboyacense, encontrándose un caso de A-C, y un caso de S-talasemia. 
Tabla 1. Porcentaje de hemoglobinopatías en San Andrés y Providencia.

\begin{tabular}{lccc}
\hline \multicolumn{1}{c}{ Isla } & $\mathbf{n}$ & Casos positivos & Porcentaje \\
\hline San Andrés & 443 & 57 & 12,8 \\
Providencia & 101 & 21 & 20,8 \\
Total & $\mathbf{5 4 4}$ & $\mathbf{7 8}$ & $\mathbf{1 4 , 3}$ \\
\hline
\end{tabular}

Tabla 2. Hemoglobinopatías halladas en San Andrés y Providencia.

\begin{tabular}{lrrrrrr}
\hline Hemoglobinopatía & \multicolumn{3}{c}{ San Andrés Providencia } & \multicolumn{2}{c}{ Total } \\
& n & \multicolumn{1}{c}{$\%$} & n & \% & n & $\%$ \\
\hline Rasgo falciforme A-S & 31 & 7,0 & 6 & 5,9 & 37 & 6,8 \\
Heterorigotos A-C & 11 & 2,5 & 5 & 5,0 & 16 & 2,9 \\
Heterocigotos S/B+Tal & 6 & 1,4 & 5 & 5,0 & 11 & 2,0 \\
Heterocigotos A/B+Tal & 4 & 0,9 & 5 & 5,0 & 9 & 1,7 \\
PHHF & 3 & 0,7 & - & - & 3 & 0,6 \\
Heterocigotos S-C & 1 & 0,2 & - & - & 1 & 0,2 \\
Homocigoto S-S & 1 & 0,2 & - & - & 1 & 0,2 \\
Total & $\mathbf{5 7}$ & $\mathbf{1 2 , 8}$ & $\mathbf{2 1}$ & $\mathbf{2 0 , 8}$ & $\mathbf{7 8}$ & $\mathbf{1 4 , 3}$ \\
\hline
\end{tabular}

Tabla 3. Frecuencia de hemoglobinopatías reportadas para diferentes regiones del país.

\begin{tabular}{llrr}
\hline Estudio & Población & n & Frecuencia \\
\hline \multirow{2}{*}{ Restrepo } & Indígena & 776 & - \\
& Negra (Chocó) & 1.422 & $13,6 \%$ \\
& Mixta-pacientes & 2.817 & $2,2 \%$ \\
Espinel & Negra (Chocó) & 1.043 & $3,8 \%$ \\
& Indígena (Chocó) & 52 & $21,2 \%$ \\
Fonseca & Altiplano cundiboyacense & 300 & $0,6 \%$ \\
\hline
\end{tabular}

Tomado de: Restrepo CA (15), Espinel A (16) y Fonseca DJ (17).

Podemos observar que en cada región la frecuencia de hemoglobinopatías es diferente y, por tanto,es recomendable no tomar los resultados de estudios regionales con el propósito de unificar este criterio para todo el país, teniendo en cuenta las características étnicas, migratorias, y geográficas diferenciales para cada región.

El departamento de San Andrés y Providencia ubicado al noroeste del territorio continental colombiano a $729 \mathrm{kms}$ de Cartagena, toma el nombre del archipiélago que lo constituye y for- ma parte de la región del Caribe. En los países centroamericanos se han realizado varios estudios de hemoglobinopatías y en éstos han sido informadas frecuencias que oscilan desde $7,4 \%$ hasta $19,7 \%(19,20)$. El Caribe es una zona geográficamente heterogénea donde el ambiente ha jugado un papel importante en la constitución genético-hematológica de las poblaciones, como resultado de la selección ambiental (20); y en donde ha tenido lugar una variedad de migraciones de caucásicos, especialmente españoles, y de población negra, que se mezclaron con los amerindios (2).

El archipiélago de San Andrés y Providencia fue descubierto por Cristobal Colón en su primer viaje en 1492 y se instalaron algunos españoles en la isla de Santa Catalina; luego, en 1641 llegaron los primeros esclavos africanos con nuevos inmigrantes de Inglaterra; en 1664 fue tomada por la tripulación del pirata Manswelt; en 1670 por el corsario inglés Morgan y en 1689 de nuevo estuvo bajo el dominio español. En 1806 fue ocupada por tropas inglesas, y de nuevo estuvo bajo el dominio español hasta 1886 cuando fue cedido al gobierno central (18). La gente del archipiélago tiene como actividad principal el turismo y el comercio.

Las hemoglobinopatías encontradas HBS y $\mathrm{Hb}$ $\mathrm{C}$, son de origen africano lo que nos indica con precisión el aporte genético de la raza negra a la actual constitución de la población, genes que llegaron desde el siglo XVII con el tráfico de esclavos. Dakar en el Atlántico africano fue el puerto más importante a través del cual se hizo el comercio de esclavos hacia el nuevo continente y desde allí, a través del Sahara, a Nigeria y Camerún (4), lo que hace suponer que la mutación de Senegal es la más ampliamente difundida. Con referencia a la ß-talasemia y la persistencia hereditaria de la hemoglobina fetal (PHHF), cuyo origen ha sido asignado a diferentes sitios geográficos, como son el Mediterráneo, Africa, Asia y Yugoeslavia $(1,7,19,20)$, no permite precisar el origen exacto de estos genes presentes en los pobladores de las islas, pero, sí podemos inferir que la heterogeneidad genética de sus pobladores es debida a la diversidad de migraciones ocurridas desde muy diversas par- 
tes del mundo hacia este lugar en épocas diferentes y que siguen ocurriendo. No podemos descartar la ocurrencia de mutaciones nuevas que han sido favorecidas por selección natural, por las condiciones geográficas propias del territorio.

\section{Conclusiones}

La hematología genética de la población del archipiélago de San Andrés y Providencia es el resultado de caracteres genéticos autóctonos, de los aportados por los inmigrantes (africanos, asiáticos y europeos) y de las condiciones ambientales de la región; dentro de este criterio se debe tener en cuenta la presión selectiva que la geografía y las condiciones climáticas han ejercido sobre la adaptabilidad de estos genes. Los inmigrantes africanos vienen de sitios donde la malaria ha operado presión de selección sobre la frecuencia de ciertas mutaciones tanto en genes $\alpha$ y $\beta$ de la hemoglobina.

Si tenemos en cuenta el criterio de un grupo de expertos de la OMS (21), que dice que desde el punto de vista estrictamente epidemiológico, toda variante hemoglobínica que tenga una frecuencia mayor del $1 \%$ es endémica para una región determinada, las variantes S y C, la B-talasemia son endémicas para San Andrés y Providencia. La vigilancia de estas patologías debe ser permanente, además, porque constituyen un problema de salud pública que debe ser tenido en cuenta dentro de los programas de diagnóstico, prevención y educación a la comunidad.

Sobre este grupo de patologías de origen genético, cuyo tratamiento no ha sido establecido aún en forma precisa, pero de implicaciones clínicas serias como es el caso de la anemia falciforme, las autoridades de salud y los médicos deben tener información de su magnitud y tomar decisiones que conduzcan a la atención del problema. En general, para las hemoglobinopatías un mayor esfuerzo debería ponérsele a la detección de heterocigotos, al diagnóstico neonatal a fin de permitir una identificación temprana y ofrecer programas de protección al individuo portador, un adecuado asesoramiento genético y educati- vo para el paciente y un manejo adecuado de los homocigotos.

\section{Agradecimientos}

Al Grupo de Química Clínica del Instituto Nacional de Salud por su colaboración en el procesamiento de biometría hematológica; al grupo que realizó el estudio de "Prevalencia del riesgo cardiovascular en la isla de San Andrés", Miguel Angel Suárez, Ralph Newbael, Gonzalo Martínez, Alejandro Giraldo y Edith Moreno, cuya muestra fue empleada para el presente estudio.

\section{Referencias}

1. Sáenz G. Hemoglobinopatías en la cuenca del Caribe. Rev Biol Trop 1988;36:361.

2. Mears J, Lachman $\mathbf{H}$, Cabbanes $\mathbf{R}$, et al. Sickle gen: its origin and diffusion from Africa. J Clin Invest 1991;68:606.

3. Flint J, Hardind R, Clegg J, et al. Why are some genetic diseases common? Hum Genet 1993;91:91.

4. Pagnier J, Mears G, Dunda-Belkhodja O, etal.Evidence for the multimeric origin of the sickle cell hemoglobin gene in Africa. Proc Natl Acad Sci USA 1984;1771.

5. Wai-Kan Y, Dozy A. Evolution of the hemogiobin $S$ and C in world populations. Science 1980;209:388.

6. Nagel L, Babry M, Pagnier J. Hematologically and genetically distinct forms of sickle cell anemia in Africa. N Engl J Med 1984;14:880-884.

7. Weatherall DJ, Clegg JB, Higss ER, et al. Hemoglobinopathies. In: Scriver CR, Beaudet AL, Sly WS, Valle D. The metabolic basis of inherited diseases. 6 th ed. McGraw Hill, 1989:2281.

8. Jack B. Hemoglobin A2 levels in health and varios hematological disorders. Am J Clin Pathol 1977;67:245.

9. Marengo-Dowe AJ. Rapid electrophoresis and quantitation of hemoglobins on cellulose acetate. J Clin Pathol 1965; 18:790.

10. Singer A, Chernoff M, et al. Studies on abnormal hemoglobins: their demostration in sickle cell anemia and other hematological disorders by means of alkali denaturation. Blood 1955;6:413.

11. Betke K, Marti HR. Estimation of small percentages of fetal haemoglobin. Nature 1959;184:1877.

12. Itano HA, Pauling LA. A rapid diagnostic test for sickle cell anemia. Blood 1959;4:66.

13. Daland GA, Castle WB. A simple and rapid method for demonstrating sickling of the red blood cells: the use of reducing agents. J Lab Clin Med 1948;33:1082.

14. Carell RW, Kay R. A simple method for the detection of unsable hemoglobins. Br J Haematol 1972;23:615. 
15. Restrepo CA. Frecuencia y distribución de las hemoglobinas anormales en Colombia. Antioquia Médica 1970;20:377.

16. Espinel A, Valenzuela N. Adaptaciones genéticas a la malaria en poblaciones afroaborígenes del Pacífico colombiano. Revista de Antropología 1991;7:119-129.

17. Fonseca DJ, Arias MC, Bustos I, et al. Estimación de la frecuencia de hemoglobinopatias en el altiplano cundiboyacense (Tesis de grado: en preparación). Bogotá: Univ. Nal. de Colombia, Facultad de Ciencias, Departamento de Biologia, 1994
18. Instituto Geográfico Agustín Codazzi. San Andrés y Providencia. Diccionario Geográfico de Colombia, tomo II, segunda edición. Bogotá, 1980:1428.

19. Sáenz G, et al. Hemoglobinas anormales y talasemias en Costa Rica, otros países de Centroamérica y Panamá. Bol Oficina Sanit Panam 1988;105:2.

20. Colombo B, Martínez G. Hemoglobinopathies. Part 2: Tropical America Clin. Haematologia 1981;10:730.

21. WHO Working Group. Hereditary anaemias: genetic basis, clinical features, diagnosis and treatment. Bulletin World Health Organ 1982;60:643. 